\title{
Sub band Fractional Fourier Transform and Application in Edge Detection
}

\author{
Weijun Lv, Enqing Chen, Lin Qi, Xin Guo
}

School of Information Engineering, Zhengzhou University, Zhengzhou, 450000, China

Keywords: FRFT, SB-FRFT, time-frequency analysis, edge detection.

\begin{abstract}
This paper proposes the concept of Sub band Fractional Fourier Transform (SB-FRFT), and extends to the 2-D sub band fractional Fourier transform. By combing the low-frequency sub band image after SB-FRFT with the traditional Sobel operator, edges of an image could be extracted more precisely. Experiments validate the proposed design method and also demonstrate the potential application of the SB-FRFT in image processing.
\end{abstract}

\section{Introduction}

As a generation of Fourier transform, FRFT is a powerful signal analyzing and processing tool which contains both time and frequency information. It can make up for the deficiency of Fourier transform in digital image processing [1-3] and pattern recognition [4-5]. But the complexity of the FRFT for high dimensional image is relatively high, it is significant to decrease that.

In digital image processing, most natural images with no obvious grain noise are decomposed into a collection of series of band-limited components called sub band. Operations can be done separately within each sub band, preventing the cross interference and the spread of noise.

Inspired by the theory of sub band decomposition, a novel method, termed 'SB-FRFT', is proposed in this paper. This method possesses the advantages of time-frequency analysis features, which is from FRFT, and the sub band properties. Meanwhile, this scheme is applied to extract the geometric shape, resulting in a perfect validation of the new algorithm.

The organizational structure of this paper is as follows: Section 2 elaborates the principles and definitions of FRFT; In Section 3, we present the definitions of 1-D and 2-D SB-FRFT; the Section 4 demonstrates the concrete application of SB-FRFT in image edge detection. Conclusions are finally drawn.

\section{Review of Fractional Fourier transform}

FRFT, is also known as angle Fourier transform [6]. Here presents the basic definition of FRFT from the view of linear integral:

$$
S_{\alpha}(u)=F^{\alpha}[s(t)]=\int_{-\infty}^{+\infty} s(t) K_{\alpha}(t, u) d t
$$

Kernel of the FRFT is:

$$
K_{\alpha}(t, u)=\left\{\begin{array}{lr}
\sqrt{\frac{1-j \cot \alpha}{2 \pi}} \exp \left(j \frac{t^{2}+u^{2}}{2} \cot \alpha-j t u \csc \alpha\right), \alpha \neq n \pi \\
\delta(t-u), & \alpha=2 n \pi \\
\delta(t+u), & \alpha=(2 n \pm 1) \pi
\end{array}\right.
$$

Where $\delta(t)$ is the impulse function? $n$ Means an integer. $\alpha=p \pi / 2$ Is the rotation angel. $p$ Denotes the order of the FRFT. 


\section{Sub band Fractional Fourier transform (SB-FRFT)}

\section{The structure of 1-D SB-FRFT}

Men et al. [7] presents the structure of two channel filter banks of FRFT domain shown in Fig.1, by using the low-pass and high-pass filters of Fourier domain to design the low-pass and high-pass filters of FRFT domain. The low-frequency component of FRFT is:

$$
\tilde{X}_{0, p}(\omega)=A_{p} \tilde{X}_{p}(\omega) \cdot \tilde{H}(\omega \csc \alpha)
$$

Where $\omega$ denotes the digital angular frequency [8] of FRFT domain, $\tilde{X}_{p}(\omega)$ is the FRFT of original signal, $\tilde{H}(\omega \csc \alpha)$ means the Fourier transformation of low-pass filter, $A_{p}$ is a constant associate with $p$.

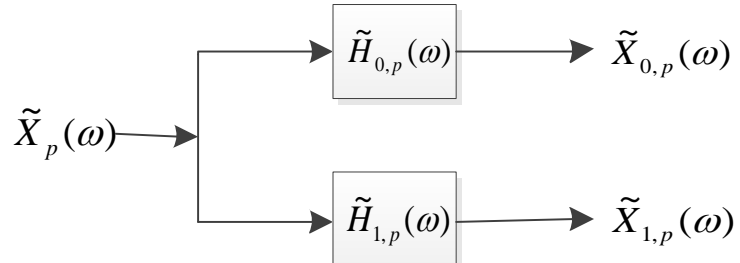

Fig.1: The structure of two-channel filter banks associated with the FRFT

Considering the low-frequency and high-frequency components as sub bands, we can design the general structure of sub band fractional Fourier transform as Fig. 2. Where $h_{0}(n)$ and $h_{1}(n)$ are the lowpass and high-pass filters of Fourier domain.

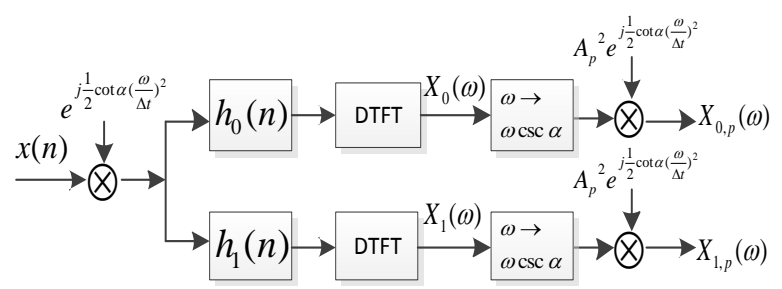

Fig.2: The general structure of SB-FRFT

Taking the low-pass and high-pass filter [9], and according to the definition of discrete FRFT [10] and Pei sampling algorithm [11], the physical structure of SB-FRFT is as Fig. 3.

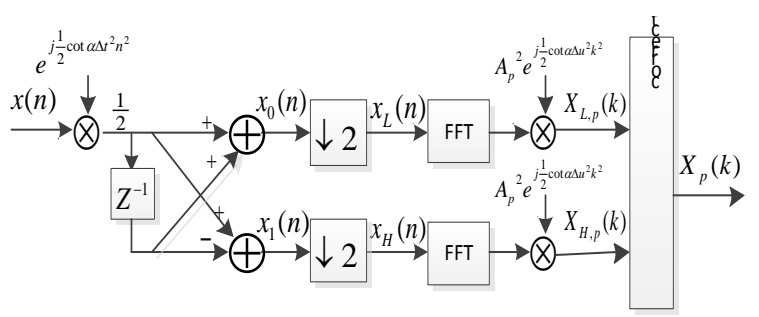

Fig.3: The physical structure of SB-FRFT

Where $X_{L, p}(k)$ and $X_{H, p}(k)$ are the low-frequency and high-frequency sub bands of FRFT domain respectively. The full-band FRFT $X_{p}(k)$ with length-N signal can be obtained by multiplying $X_{L, p}(k)$ and $X_{H, p}(k)$ with correction factors $1+W_{N}^{k}$ and $1-W_{N}^{k}$, with $W_{N}$ denoting $e^{-j 2 \pi / N}$.

$$
X_{p}(k)=\left(1+W_{N}^{k}\right) X_{L, p}(k)+\left(1-W_{N}^{k}\right) X_{H, p}(k)
$$

If the signal is known having negligible energy in the frequency $f \in\left(f_{s} / 4, f_{s} / 2\right)$ (with $f_{s}$ denoting the sampling frequency), (4) is approximated for calculating only the low-frequency band.

$$
\hat{X}_{p}(k) \approx\left(1+W_{N}^{k}\right) X_{L, p}(k)
$$

Two types of approximation errors occur in the process (5): a linear distortion according to nonconstant frequency response in the band of interest (low-pass), and aliasing due to non-zero components in the neglected band.

The decomposition process in Fig. 3 can be repeated again and again to obtain smaller and smaller sub bands. 
The structure of 2-D SB-FRFT

The computation of 2-D SB-FRFT using a separable sub band decomposition scheme in both spatial variables is presented in Fig. 4 . We can obtain the 2-D SB-FRFT:

$$
\begin{aligned}
X_{p 1, p 2}(k, l)= & \left(1+W_{N}^{k}\right)\left(1+W_{N}^{\prime}\right) X_{L L, p l, p 2}(k, l)+\left(1+W_{N}^{k}\right)\left(1-W_{N}^{\prime}\right) X_{L H, p 1, p 2}(k, l) \\
& +\left(1-W_{N}^{k}\right)\left(1+W_{N}^{\prime}\right) X_{H t, p l, p 2}(k, l)+\left(1-W_{N}^{k}\right)\left(1-W_{N}^{\prime}\right) X_{H H, p l, p 2}(k, l)
\end{aligned}
$$

Where $X_{L L, p_{1}, p_{2}}(k, l) X_{L H, p_{1}, p_{2}}(k, l) X_{\mathrm{HL}, p_{1}, p_{2}}(k, l)$ and $X_{H H, p_{1}, p_{2}}(k, l)$ are Low-Low sub band, Low-High sub band, High-Low sub band and High-High sub band of FRFT domain respectively. $\left(1 \pm W_{N}^{k}\right)\left(1 \pm W_{N}^{l}\right)$ Mean the correction factors.

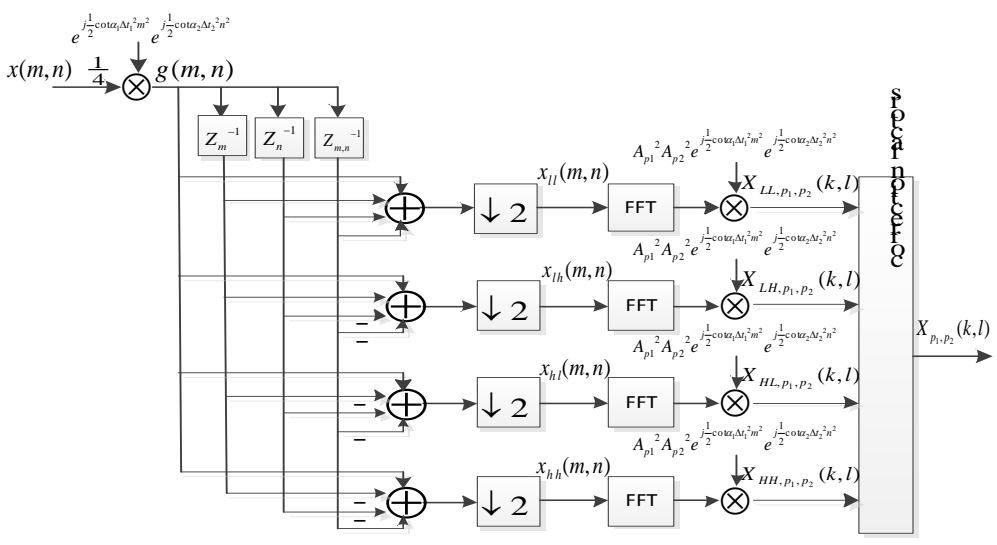

Fig.4: 2-D SB-FRFT calculation

If the signal has little energy content above, $k, l \in(0, N / 4)$ the aliasing components in (6) can be neglected, the approximate 2-D SB-FRFT of order $p_{1}, p_{2}$ can be given as:

$$
\hat{X}_{p 1, p 2}(k, l) \approx\left(1+W_{N}^{k}\right)\left(1+W_{N}^{l}\right) X_{L L, p 1, p 2}(k, l)
$$

\section{Application in edge detection}

The current image edge detection methods are mainly differential operator technique and high-pass filtration. Among these methods, the most primitive of the differential and gradient edge detection methods are complex and the effect are not satisfactory.

To extract edges with less noised or blurred cases, we here combine the 2-D SB-FRFT and the traditional Sobel operator to extract edges as the diagram in Fig. 5. The 2-D SB-FRFT decomposes an image into different-frequency sub bands of FRFT domain, and we use Sobel operator [12] to deal with the low-frequency sub band image, resulting in a gradient image. After looking for the local maximum in the gradient image and threshold processing, we get the final edge image.

In this study, the 'Cameraman' image with Gaussian white noise is taken as the original image. First, we use the traditional edge detection operators, Sobel, Canny operator [13-14] and wavelet method [15], to extract edges. The results are shown in Fig. 6.

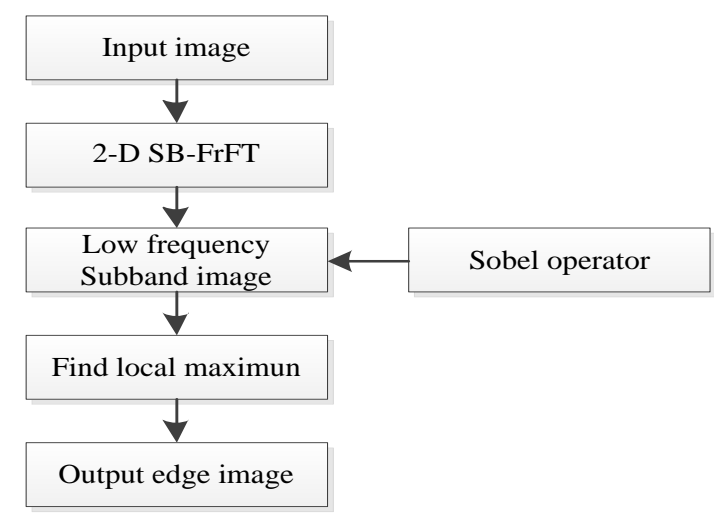

Fig.5: The diagram of the proposed edge extraction approach 


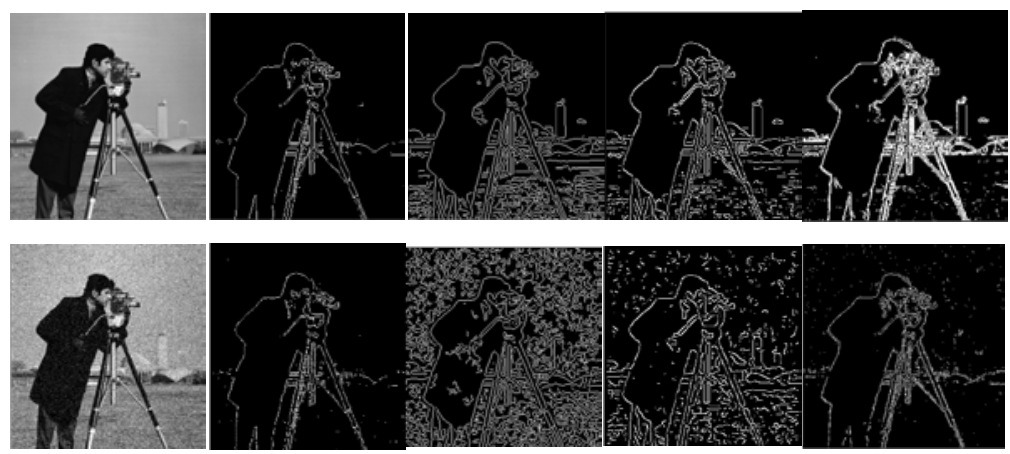

Fig.6: The extracted edges of the different method:

(a) Original Cameraman image. (b) Noisy Cameraman image.

(c)- (d) Edges based on the Sobel method (e)-(f) Edges based on the canny method.

(g)- (h) Edges based on the wavelet transform method (i)-(j) Edges based on the proposed method

From the Fig.6, we can see that the traditional operators will detect all the noise points and blur the edge details of the images. Even the detection results of the classical canny operator is not satisfactory either. It is because that the traditional edge detection operators mostly use the differences of the neighborhood gray values. However, when the image adulterates lots of noise signals, there are gray value differences between white noise and image signal, which can be easily detected. The Gaussian smoothing filters cannot perfectly eliminate the influence of the noise, which leads to the poor detection effect of the classical operators. Wavelet method has a better effect than traditional differential methods in treating images that with noise.

In Fig. 6, (i) and (j) show the edge detection results of the proposed approach. Compared with other methods, low-frequency sub band image of 2-D SB-FRFT has a better suppression effect for Gaussian white noise and texture information. This makes the effect of edge detection using Sobel operator more outstanding.

Generally, the noise and texture information are located in the high frequency part. The lowfrequency information is mainly stored as the low-frequency sub band image after 2-D SB-FRFT. In this way, the influence of noise and complex texture can be effectively avoided. Because the high frequency is weakened in the low-frequency sub band image, we can get a stronger gradient image when treating low-frequency sub band image with Sobel operator. After finding the local maximum points in the gradient image and threshold processing, we get the better edge image.

Several attempts have been made in the literature to propose an objective measure for the performance of an edge detector. While there is no consensus and each of these measures has their limitations, one of the most recognized measure is the Pratt's Figure of Merit (FOM) [16], whose definition is based on the combination of three factors: non detection of true edges, detection of false edges and edge delocalization error, which is defined as

$$
F=\frac{1}{\max \left(N_{d}, N_{o}\right)} \sum_{i=1}^{N_{d}} \frac{1}{1+\alpha d_{i}^{2}}
$$

Where $N_{d}$ the number of detected edge points $N_{o}$ means the number of original edge points, $\alpha$ is a calibration constant, generally fixed at $1 / 9$. Hence, the output from Pratt's Figure of Merit is fidelity function ranging from 0 to 1 , where 1 is a perfect edge detector. $d_{i}$ Denotes the Euclidian distance between an edge pixel (detected edge point) denoted by index $i$ in the distorted image and the corresponding one (original edge point) in the reference edge map.

We computed the Pratt's Figure of Merit using the image shown in Fig.7. The criteria need a trueedge image for comparison. Since in the case the exact edge map is not known, it is computed using the classical canny algorithm on the image without noise. The results reported in the following Table confirms the visual impression in our tests. The proposed approach is shown to yield higher values for the FOM. 

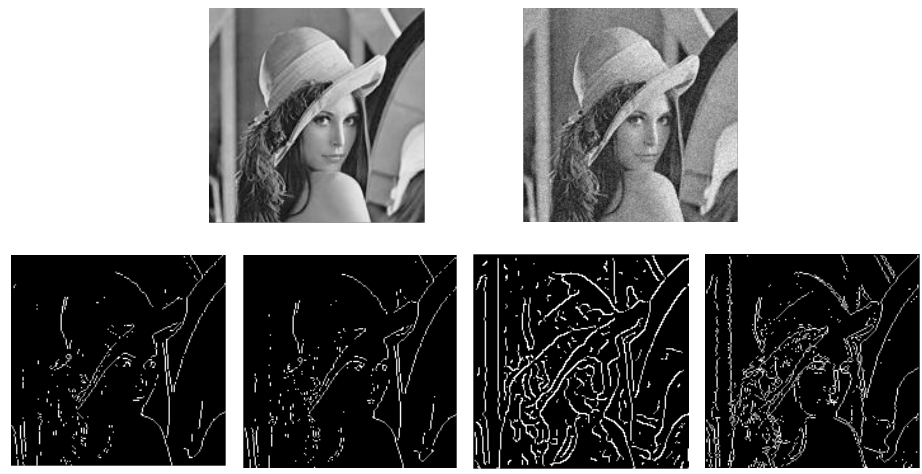

Fig.7: Results of edge detection methods.

(a) The original test image. (b) Noisy image. (c) The detected edges of Soble detector. (d) The extracted edges of Prewitt detector. (e) The detected edges of wavelet method. (f) The extracted edges of the proposed method.

We compare the proposed approach with other edge detectors, such as Sobel, Prewitt and wavelet transform, their FOM values are shown in Table 1.

Table 1 Pratt's FOM for the image of Fig. 6

\begin{tabular}{c|c|c|c|c}
\hline & Sobel & Prewitt & Wavelet method & The proposed approach \\
\hline FOM & 0.4595 & 0.4404 & 0.8440 & 0.8992 \\
\hline
\end{tabular}

The criterion values show that the proposed approach is quantitatively better than other methods. The proposed approach extracts more complete edges and has better localization performance.

\section{Conclusions}

In this study, the structure of the 1-D and 2-D SB-FRFT is proposed, in which the 2-D structure is viewed from the image processing. According to the structure of the 2-D SB-FRFT, we can get four sub bands of different-frequency of FRFT domain. When multiplying corresponding factors, we can get the FRFT of the original image. This paper states the specific application in image edge detection using 2-D SB-FRFT, and obtains satisfactory edge detection results, especially for the images with Gaussian white noise. We can conclude that SB-FRFT is valuable in image edge detection. What is more, it will get more attentions and applications in the fields of image processing.

\section{Acknowledgment}

This work is supported by the National Natural Science Foundation of China, No. 61331021, and is partly supported by the National Natural Science Foundation of China, No. 61201251 and No. 61210005.

\section{References}

[1] Rafael C. Gonzalez, Richard E. Woods. Digital Image Processing. (Person Education, 2003).

[2] Soo-Chang Pei, Tianjin Ding. Two-Dimensional Affine Generalized Fractional Fourier Transform. IEEE Trans ON SP, APRIL 2001, vol. 49 (2001) No.3, p. 878.

[3] A. Shahin, M. A. Kutay and H. M. Ozaktas, Non-separable two-dimensional fractional Fourier Transform, Applied Optics, vol. 37 (1988) No.23, p.5444.

[4] Lin Qi, Enqing Chen, Xiaomin Mu, and Ling Guan. Recognition Human Emotional State Based on the 2D-FrFT and FLDA. Image and Signal Processing, CISP '09.2nd International Congress, 2009.

[5] Lei Gao, Lin Qi, Enqing Chen, Xiaomin Mu, and Ling Guan. Recognition Human Emotional State Based on the Phase Information of the Two Dimensional Fractional Fourier 
Transform. In: PCM 2010, Part II, LNCS 6298, 2010, p. 696.

[6] H. M. Ozaktas, O. Arikan, M. A. Kutay. Digital Computation of the Fractional Fourier Transformation. IEEE Trans on SP, vol. 44 (1996) No.9, p. 2441.

[7] Xiang Yi Meng, Ran Tao, Yue Wang. The fractional Fourier domain analysis of two channel filter banks. Chinese Journal of Electronics. vol. 36 (2008) No.5, p. 919.

[8] Xiang Yi Meng, Ran Tao, Yue Wang. Fractional Fourier domain analysis of decimation and interpolation. Science in China: Series F, vol. 50 (2007) No.4, p.521.

[9] A. Hossen and U. Heute. Two-dimensional sub band transforms: theory and applications. IEE Proc.-Vis. Image Signal Process. vol. 151 (2004) No.5, p. 389.

[10] Erseghe T, et al. Unified fractional Fourier transform and sampling theorem [J]. IEEE Trans. on SP Letters, vol. 47 (1999) No.12, p.3419.

[11] Soo-Chang Pei, Jian-jiun Ding. Closed-form discrete fractional and affine Fourier transform. IEEE Trans. on SP, vol. 48 (2000) No.5, p.1338.

[12] I. Sobel and G. Feldman, A 3x3 isotropic gradient operator for image processing, presented at a talk at the Stanford Artificial Intelligence Project, 1968.

[13] J. Canny. A computational approach to edge detection. IEEE Trans. Pattern. Machine Intel. vol. 6 (1986) No.6, p. 679.

[14] W. McIlhagga, the Canny edge detector revisited, International Journal of Computer Vision, vol. 91 (2011) No.3, p. 251.

[15] Erseghe T, et al. Unified fractional Fourier transform and sampling theorem. IEEE Trans. on SP Letters, vol. 47 (1999) No.12, p. 3419.

[16] Weifang Ma, Caixia Deng, An improved wavelet multi-scale edge detection algorithm. Proceeding of the International conference on Wavelet Analysis and Pattern Recognition, 2012, p. 302.

[17] W. K. Pratt, Digital image processing. (New York: Wiley-Inter-science, 1978). 